\title{
A 3-D Stochastic FDTD Model of Electromagnetic Wave Propagation in Magnetized Ionosphere Plasma
}

\author{
Bach T. Nguyen, Student Member, IEEE, Cynthia Furse, Fellow, IEEE, and \\ Jamesina J. Simpson, Senior Member, IEEE
}

\begin{abstract}
A stochastic finite-difference time-domain (S-FDTD) algorithm is presented for electromagnetic-wave propagation in anisotropic magnetized plasma. This new algorithm efficiently calculates in a single simulation not only the mean electromagnetic field values, but also their variance as caused by the variability or uncertainty of the electron and ion content of the ionosphere. The structure of the ionosphere is often too variable and uncertain for electromagnetic-wave propagation problems to be solved using a deterministic formulation, particularly during space weather events. For these cases, the S-FDTD ionospheric plasma algorithm will serve as an important tool. For example, it could be used to determine the confidence level at which a communications or remote sensing or radar system will operate as expected under abnormal ionospheric conditions.
\end{abstract}

Index Terms-Earth, electromagnetic wave propagation, finite-difference time-domain (FDTD), ionosphere, magnetized cold plasma, statistics, stochastic finite-difference time-domain (S-FDTD) method, uncertainty, variance.

\section{INTRODUCTION}

$\mathbf{C}$ OMMUNICATIONS, surveillance, and navigation capabilities rely heavily on accurate knowledge of electromagnetic (EM) signal propagation characteristics through, and reflected by, the Earth's ionosphere. Satellite communications, over-the-horizon radar, and target direction finding are a few example applications. Poor understanding of either the ionospheric state or the complete signal propagation characteristics through the ionosphere can negatively affect the performance of these applications. For example, inaccurate signal predictions may lead to erroneous target identification and coordinate estimation [1].

It is crucial for the performance of many of these systems to have knowledge of not just the general (average) structure of the ionosphere, but also its variability (or uncertainty). For example, the variability of the ionosphere strongly effects transionospheric radio propagation. The irregularities in the electron density distribution cause phase and amplitude scintillation. We note that there are now efforts to add variance electron and

Manuscript received December 30, 2013; revised August 18, 2014; accepted September 20, 2014. Date of publication October 30, 2014; date of current version December 31, 2014. This work is supported by National Science Foundation CAREER Award grant 0955404.

The authors are with the Department of Electrical and Computer Engineering, University of Utah, Salt Lake City, UT 84112 USA (e-mail: jamesina.simpson@utah.edu).

Color versions of one or more of the figures in this paper are available online at http://ieeexplore.ieee.org.

Digital Object Identifier 10.1109/TAP.2014.2365824 ion information to the International Reference Ionosphere (IRI) dataset [2]-[9].

The variability of the ionosphere renders many propagation problems too complex to be solved using a deterministic formulation. The structure of the ionosphere can depend not only on the altitude, time of day, and season, but also on the latitude longitude, sunspot cycle, and occurrence of space weather events. A useful approach to such a highly complex problem as EM-wave propagation in the ionosphere is to consider it as a random medium problem.

Numerical EM techniques, however, typically use only average (mean) values of the constitutive parameters of the materials and then solve for expected (mean) electric and magnetic fields. The Monte Carlo method is a well-established and widely used brute force technique for evaluating random medium problems via multiple realizations [10]. Depending on the nature of the statistical correlation, a random medium problem may require tens or hundreds of thousands of realizations. This yields an extremely inefficient brute force approach, particularly for two-dimensional and three-dimensional problems, and is therefore rarely used in EM modeling.

The finite-different time-domain (FDTD) method [11], [12] has been demonstrated as a robust and efficient computational electromagnetic technique for Earth-ionosphere wave propagation problems (e.g., [13]-[19]). Several techniques have been proposed recently to solve uncertainty quantification problems involving the FDTD solution to Maxwell's equations. Stochastic FDTD (S-FDTD) is an efficient formulation that runs the ensemble averages in a single realization scheme [20], [21]. Reference [20] provides a direct estimate of both the mean and variance of the electromagnetic fields within layered biological media at every point in space and time. The advantage of this method is that it requires only about twice as much computer simulation time and memory as a traditional FDTD simulation, regardless of the number of random variables. On the other hand, its limitation is that it can only bound the field variances according to a best estimate approximation for the cross-correlation coefficients.

The approach in [21] proposes a single-realization scheme to obtain the ensemble average of the scattered fields. The advantage of the approach of [21] is that it makes use of an iterative technique to reformulate a multiplicative noise into an additive noise. However, the limitation of this algorithm is that it must meet the condition of a weakly scattering random medium, wherein the deviation from the mean electrical material values is small. 
Another approach [22], [23] makes use of the generalized polynomial chaos (gPC) method, which is an extension of homogeneous chaos introduced by Wiener in 1938. The gPC expands the time-domain electric and magnetic fields in terms of orthogonal polynomial basis functions of the uncertain variables. The infinite sum of the polynomial chaos expansion is truncated to a finite number of terms $P$ of orthogonal basis functions. The number of terms $P$ is given by $P+1=(n+$ $d) ! /(n ! d !)$, where $d$ is the highest polynomial order in the expansion and $n$ is number of random variables. It follows that $P$ grows very quickly with the dimension and order of the decomposition. In general, the gPC method increases memory consumption by a factor $P+1$ and the simulation time proportional to $(P+1)^{2}$. The gPC results, however, typically converge significantly faster than the Monte Carlo method in a number of applications. It is also a mathematically robust method. However, the method has an inherent limitation. It can handle only a limited number of uncertain inputs. For large numbers of random variables, polynomial chaos becomes very computationally expensive, and Monte Carlo methods are typically more feasible.

In summary, each of the above approaches has its own strengths and limitations. Given the fact that the ionosphere content can vary even up to $100 \%$ or more, the S-FDTD method proposed in [20] and the gPC method are good candidates for electromagnetic-wave propagation modeling in ionosphere plasma.

An FDTD fully three-dimensional (3-D) anisotropic magnetized plasma model is reported in [17] and [18] for application to electromagnetic-wave propagation in the ionosphere. This model solves Maxwell's equations coupled to current equations derived from the Lorentz equation of motion. By providing 3-D solutions, it yields the ability to simulate Faraday rotation and the complete 3-D spatial variations of the magnetized cold plasma. The accuracy, stability, and formulation of this method is fully described in [17, Sections I and II]. In [19], the Cartesian magnetized plasma ionosphere algorithm of [17] is extended to the global latitude-longitude FDTD model of the Earth-ionosphere waveguide.

In the algorithm of [17], the governing stochastic equations take the form of a large, complex matrix. As a result, the complexity of the physical model presents a computational challenge. If the gPC method is applied for variation analysis of uncertainty effects on the ionospheric plasma electromagnetic field, the derivation of the explicit equations for the gPC coefficients may be very difficult, or even impossible to obtain.

Comparing all the above strengths and weaknesses of the different stochastic modeling approaches, currently the S-FDTD method presented in [20] is the only suitable method for modeling large-scale, complex problems of Earth-ionosphere wave propagation. Therefore, in this paper, we extend the methodology of Maxwell's equations S-FDTD [20] to Maxwell's equations coupled to the Lorentz equation of motion as in the 3-D FDTD magnetized plasma algorithm of [17] and [18]. We also propose an initial method for determining a good approximation for the cross correlation coefficients.

The new S-FDTD ionosphere plasma model presented here has broad potential applicability. The ability to determine not only the mean values of the ionospheric EM fields but also their variance will, for example, provide the capability of determining the confidence level that a communications/remote sensing/radar system will operate as expected under abnormal ionospheric conditions. It may also be useful in a wide variety of geophysical studies.

\section{Methodology}

\section{A. Governing Equations}

The plasma algorithm methodology presented in [17] is identical for each electron, positive and negative ion species within the ionosphere. Since the effect of ions on EM-wave propagation may sometimes be neglected, and to simplify the presentation of the S-FDTD ionosphere algorithm in this paper, we will consider a simplified case of a plasma comprised of only electrons. The cold plasma is characterized by a free-space permittivity and a free-space permeability that is biased by an applied magnetic field $B$.

The magnetized cold plasma governing equations are cast in terms of Maxwell's equations coupled to current equations derived from the Lorentz equation of motion. The resulting whole governing equation set is given by

$$
\begin{aligned}
\nabla \times \mathbf{E} & =-\mu_{0} \frac{\partial \mathbf{H}}{\partial t} \\
\nabla \times \mathbf{H} & =\epsilon_{0} \frac{\partial \mathbf{E}}{\partial t}+\mathbf{J}_{e} \\
\frac{\partial \mathbf{J}_{e}}{\partial t}+v_{e} \mathbf{J}_{e} & =\epsilon_{0} \omega_{P e}^{2} \mathbf{E}+\boldsymbol{\omega}_{C e} \times \mathbf{J}_{e} .
\end{aligned}
$$

Here, $v_{e}, \mathbf{J}_{e}$, and $\omega_{P e}$ are the collision frequency, the current density, and the plasma frequency of electrons, respectively. The plasma frequency is given by

$$
\omega_{P e}=\sqrt{\frac{q_{e}^{2} n_{e}}{\epsilon_{0} m_{e}}} .
$$

It should be noted that the electron plasma frequency is a function of the electron density $n_{e}$. Ionosphere electron densities vary in a complex manner as a function of location and time. Thus, we consider the electron density as a random variable with its own statistical variation. This variability in the electron density causes variability in the EM fields, which will also be treated as random variables.

The current density can be written using Cartesian coordinates as

$$
\mathbf{J}_{e}=J_{e x} \hat{x}+J_{e x} \hat{y}+J_{e x} \hat{z} .
$$

$\boldsymbol{\omega}_{C e}$ is the cyclotron frequency of the electrons given by $\boldsymbol{\omega}_{C e}=$ $\left|q_{e}\right| \mathbf{B} / m_{e}$ with a Cartesian coordinate expressions as

$$
\boldsymbol{\omega}_{C e}=\omega_{C e x} \hat{x}+\omega_{C e x} \hat{y}+\omega_{C e x} \hat{z} .
$$

Note also that the cyclotron frequency is a function of the applied magnetic field, which is taken to be constant. Thus, the cross-product terms in (3) makes the plasma anisotropic so that the wave behavior depends on its propagation direction relative to the direction of the magnetic field.

\section{B. Mean Field Equations}

For the S-FDTD derivation, there are initially three stochastic equations (1), (2), and (3) that for Cartesian coordinates contain ten random variables for the 3-D case: $E_{x}, E_{y}, E_{z}, H_{x}, H_{y}, H_{z}$, 
$J_{e x}, J_{e y}, J_{e z}$, and $\omega_{P e}$. By using the delta method [24], Smith and Furse demonstrated that the average (or expected) fields can be found by solving the field equations using the mean or averages of the variables [20]. Analogously, we find for the case of the S-FDTD magnetized cold plasma model that the equations for the mean values of the EM fields and current densities are also equal to those of the regular 3-D FDTD magnetized cold plasma model equations presented in [17] and [18] (corresponding for the $x$-component case to (7), (8), and (9) of [18]), as follows:

$$
\begin{aligned}
\left.H_{x}\right|_{i, j+1 / 2, k+1 / 2} ^{n+1 / 2} & \left.H_{x}\right|_{i, j+1 / 2, k+1 / 2} ^{n-1 / 2}+\frac{\Delta t}{\mu_{0}}\left[\frac{\left.E_{y}\right|_{i, j+1 / 2, k+1} ^{n}-\left.E_{y}\right|_{i, j+1 / 2, k} ^{n}}{\Delta z}\right. \\
& \left.-\frac{\left.E_{z}\right|_{i, j+1, k+1 / 2} ^{n}-\left.E_{z}\right|_{i, j, k+1 / 2} ^{n}}{\Delta y}\right] \\
\left.E_{x}\right|_{i+1 / 2, j, k} ^{n+1}+\left.\frac{1}{2} \widehat{J}_{e x}\right|_{i+1 / 2, j, k} ^{n+1} & \left.E_{x}\right|_{i+1 / 2, j, k} ^{n} \\
& -\left.\frac{1}{2} \widehat{J}_{e x}\right|_{i+1 / 2, j, k} ^{n}+\frac{(\Delta t)^{2}}{\epsilon_{0} \mu_{0} \Delta z}\left(\frac{\Delta \widehat{H}_{z}}{\Delta y}\right)_{i+1 / 2, j, k}^{n+1 / 2} \\
& -\frac{(\Delta t)^{2}}{\epsilon_{0} \mu_{0} \Delta y}\left(\frac{\Delta \widehat{H}_{y}}{\Delta z}\right)_{i+1 / 2, j, k}^{n+1 / 2} \\
& -\left.\frac{\left(\omega_{P e} \Delta t\right)^{2}}{2} E_{x}\right|_{i+1 / 2, j, k} ^{n+1}+\left.\left(1+\frac{v_{e} \Delta t}{2}\right)^{n} \widehat{J}_{e x}\right|_{i+1 / 2, j, k} ^{n+1} \\
& +\left.\frac{\omega_{c e z} \Delta t}{2} \widehat{J}_{e y}\right|_{i+1 / 2, j, k} ^{n+1}-\left.\frac{\omega_{c e y} \Delta t}{2} \widehat{J}_{e z}\right|_{i+1 / 2, j, k} ^{n+1} \\
= & \left.\frac{\left(\omega_{P e} \Delta t\right)^{2}}{2} E_{x}\right|_{i+1 / 2, j, k} ^{n}+\left.\left(1-\frac{v_{e} \Delta t}{2}\right) \widehat{J}_{e x}\right|_{i+1 / 2, j, k} ^{n} \\
& -\left.\frac{\omega_{c e z} \Delta t}{2} \widehat{J}_{e y}\right|_{i+1 / 2, j, k} ^{n}+\left.\frac{\omega_{c e y} \Delta t}{2} \widehat{J}_{e z}\right|_{i+1 / 2, j, k} ^{n}
\end{aligned}
$$

Thus, the mean EM field and current density values are found by using the mean plasma frequency of $\omega_{P e}$, or equivalently, the mean of electron density $n_{e}$. Note that the magnetic fields and current densities are scaled such that $\widehat{H}_{u}=\left(\mu_{0} \Delta u / \Delta t\right) H_{u}[u=x, y, z]$ and $\widehat{J}=\left(\Delta t / \epsilon_{0}\right) J$ to avoid instabilities and inaccuracies caused by the large iteration coefficients. Note also for brevity, we use the notation

$$
\left(\frac{\Delta \widehat{H}_{z}}{\Delta y}\right)_{i+1 / 2, j, k}^{n+1 / 2}=\frac{\left.\widehat{H}_{z}\right|_{i+1 / 2, j+1 / 2, k} ^{n+1 / 2}-\left.\widehat{H}_{z}\right|_{i+1 / 2, j-1 / 2, k} ^{n+1 / 2}}{\Delta y} .
$$

Further, the mean calculations presented here are a first-order accurate single-realization ensemble average scheme. The accuracy may be improved in the future by developing higher order methods that include the higher order terms from the Taylor series. However, the validation test cases presented in this paper have demonstrated that the first-order accurate scheme is a very good approximation for magnetized cold plasma model. Moreover, the primary purpose of S-FDTD is to quantify the variance of the fields rather than the mean field values.

\section{Variance Field Equations}

When solving only Maxwell's equations, the variance field equations can be solved separately from the mean field equations no matter the dimensionality of the problem [25]. However, in the 3-D magnetized cold plasma model, the Lorentz (3) is coupled to the Maxwell (2), which leads to a complicated but linear system, as can be seen in (8) and (9). As a result, the electric field and current density variances must be computed simultaneously. When variance equations are derived, covariances are needed of the $E, H$ fields and current density $J_{e}$ in both time and space. The equations also relate the electric field to the plasma frequency of the ionosphere, resulting in additional covariance terms between the electric field and the plasma frequency. For the S-FDTD method, a critical step is to approximate the correlation coefficients.

As for the 3-D Maxwell's equations S-FDTD methodology of [25], for the 3-D S-FDTD magnetized cold plasma algorithm, the magnetic fields, electric fields, and current densities are highly correlated to each other. As such, the correlation coefficients of the $E$ and $H$ fields and current density $J_{e}$ may be approximated as 1 . The approximation of the remaining correlation coefficients between the electric field and the plasma frequency will control the accuracy of the algorithm.

In order to derive the standard deviation (or variance) equations, we must take the variance of (7), (8), and (9). This step results in two cases as described below:

Case 1: If a function is formed by the sum of multiple variables ((7) and (8)), its variance is

$$
\begin{aligned}
& \sigma^{2}\left\{\sum_{i=1}^{n} a_{i} X_{i}\right\} \\
& =\sum_{i=1}^{n} a_{i}^{2} \sigma^{2}\left\{X_{i}\right\}+2 \sum_{1 \leq i<j \leq n} \sum_{i} a_{j} \rho_{X_{i}, X_{j}} \sigma\left\{X_{i}\right\} \sigma\left\{X_{j}\right\} .
\end{aligned}
$$

Here, $\rho_{X_{i}, X_{j}}$ is the correlation coefficient $\left(-1 \leq \rho_{X_{i}, X_{j}} \leq\right.$ $1)$. The closer this coefficient is to zero, the more independent the terms are from each other. If the correlation coefficients $\rho_{X_{i}, X_{j}}(1 \leq i<j \leq n)=1$, we obtain

$$
\begin{aligned}
\sigma^{2}\left\{\sum_{i=1}^{n} a_{i} X_{i}\right\} & =\left[\sum_{i=1}^{n} a_{i} \sigma\left\{X_{i}\right\}\right]^{2} \\
\text { or } \sigma\left\{\sum_{i=1}^{n} a_{i} X_{i}\right\} & =\sum_{i=1}^{n} a_{i} \sigma\left\{X_{i}\right\} .
\end{aligned}
$$

Case 2: If a function is formed by the product of multiple variables $((9))$, its variance is solved by using the delta method [24], as follows:

$$
\begin{array}{r}
\sigma^{2}\left\{f\left(X_{1}, X_{2}, \ldots, X_{m}\right) g\left(X_{m+1}, X_{m+2}, \ldots, X_{m+2}\right)\right\} \\
=\left.\sum_{i=1}^{m+n} \sum_{j=1}^{m+n} \frac{\partial(f g)}{\partial X_{i}} \frac{\partial(f g)}{\partial X_{j}}\right|_{\mu_{X_{1}}, \ldots, \mu_{X_{m+n}}} \operatorname{Cov}\left(X_{i}, X_{j}\right) .
\end{array}
$$

Equations (12) and (13) will be used in the derivation of the variance equations (14), (15), and (16).

Now the S-FDTD plasma update equations will be derived, starting with the $H$-fields. For illustration purposes, only the 
update equation for the $x$-component of the $H$-field will be derived. We start by rearranging (7) and taking its variance. This yields

$$
\begin{array}{r}
\sigma^{2}\left\{\left.H_{x}\right|_{i, j+1 / 2, k+1 / 2} ^{n+1 / 2}-\left.H_{x}\right|_{i, j+1 / 2, k+1 / 2} ^{n-1 / 2}\right\} \\
=\sigma^{2}\left\{\frac { \Delta t } { \mu _ { 0 } } \left[\frac{\left.E_{y}\right|_{i, j+1 / 2, k+1} ^{n}-\left.E_{y}\right|_{i, j+1 / 2, k} ^{n}}{\Delta z}\right.\right. \\
\left.\left.-\frac{\left.E_{z}\right|_{i, j+1, k+1 / 2} ^{n}-\left.E_{z}\right|_{i, j, k+1 / 2} ^{n}}{\Delta y}\right]\right\} .
\end{array}
$$

Applying the following approximations:

$$
\begin{aligned}
\rho_{\left.H_{x}\right|_{i, j+1 / 2, k+1 / 2} ^{n+1 / 2},\left.H_{x}\right|_{i, j+1 / 2, k+1 / 2} ^{n-1 / 2}} & \approx \rho_{\left.E_{y}\right|_{i, j+1 / 2, k+1} ^{n},\left.E_{y}\right|_{i, j+1 / 2, k} ^{n}} \\
& \approx \rho_{\left.E_{z}\right|_{i, j+1, k+1 / 2} ^{n},\left.E_{z}\right|_{i, j, k+1 / 2} ^{n}} \approx 1 \\
\rho_{\left.E_{y}\right|_{i, j+1 / 2, k+1} ^{n},\left.E_{z}\right|_{i, j+1, k+1 / 2} ^{n}} & \approx \rho_{\left.E_{y}\right|_{i, j+1 / 2, k+1} ^{n},\left.E_{z}\right|_{i, j, k+1 / 2} ^{n}} \\
& \approx \rho_{\left.E_{y}\right|_{i, j+1 / 2, k} ^{n},\left.E_{z}\right|_{i, j, k+1 / 2} ^{n}}^{n} \\
& \approx \rho_{\left.E_{y}\right|_{i, j+1 / 2, k} ^{n},\left.E_{z}\right|_{i, j, k+1 / 2} ^{n}}^{n} \approx 1
\end{aligned}
$$

and applying (12) and rearranging, the resulting equation from taking the variance of (7) yields

$$
\begin{aligned}
\sigma & \left\{\left.H_{x}\right|_{i, j+1 / 2, k+1 / 2} ^{n+1 / 2}\right\} \\
= & \sigma\left\{\left.H_{x}\right|_{i, j+1 / 2, k+1 / 2} ^{n-1 / 2}\right\} \\
& +\frac{\Delta t}{\mu_{0}}\left[\frac{\sigma\left\{\left.E_{y}\right|_{i, j+1 / 2, k+1} ^{n}\right\}-\sigma\left\{\left.E_{y}\right|_{i, j+1 / 2, k} ^{n}\right\}}{\Delta z}\right. \\
& \left.-\frac{\sigma\left\{\left.E_{z}\right|_{i, j+1, k+1 / 2} ^{n}\right\}-\sigma\left\{\left.E_{z}\right|_{i, j, k+1 / 2} ^{n}\right\}}{\Delta y}\right] .
\end{aligned}
$$

Next, we will work with (8). Again, for illustration purposes, we will only drive the update equations for the $x$-component of the fields. Rearranging (8) and taking its variance yields

$$
\left.\begin{array}{rl}
\sigma^{2}\left\{\left.E_{x}\right|_{i+1 / 2, j, k} ^{n+1}-\left.E_{x}\right|_{i+1 / 2, j, k} ^{n}\right. & \\
+ & \left.\left.\frac{1}{2} \widehat{J}_{e x}\right|_{i+1 / 2, j, k} ^{n+1}+\left.\frac{1}{2} \widehat{J}_{e x}\right|_{i+1 / 2, j, k} ^{n}\right\} \\
= & \sigma^{2}\left\{\frac{(\Delta t)^{2}}{\epsilon_{0} \mu_{0} \Delta z}\left(\frac{\Delta \widehat{H}_{z}}{\Delta y}\right)_{i+1 / 2, j, k}^{n+1 / 2}\right. \\
& -\frac{(\Delta t)^{2}}{\epsilon_{0} \mu_{0} \Delta y}\left(\frac{\Delta \widehat{H}_{y}}{\Delta z}\right)_{i+1 / 2, j, k}^{n+1 / 2}
\end{array}\right\} .
$$

Applying the following approximations:

$$
\begin{aligned}
\rho_{\left.E_{x}\right|_{i+1 / 2, j, k} ^{n+1},\left.E_{x}\right|_{i+1 / 2, j, k} ^{n+1}} & \approx \rho_{\widehat{J}_{e x}{ }_{i+1 / 2, j, k}^{n+1},\left.\widehat{J}_{e x}\right|_{i+1 / 2, j, k} ^{n}} \\
& \approx \rho\left(\Delta \widehat{H}_{z} / \Delta y\right)_{i+1 / 2, j, k}^{n+1 / 2} \\
& \approx 1 \\
\rho_{\left.E_{x}\right|_{i+1 / 2, j, k} ^{n+1},\left.\widehat{J}_{e x}\right|_{i+1 / 2, j, k} ^{n+1}} & \approx \rho_{\left.E_{x}\right|_{i+1 / 2, j, k},\left.\widehat{J}_{e x}\right|_{i+1 / 2, j, k} ^{n+1}} \\
& \approx \rho_{\left.E_{x}\right|_{i+1 / 2, j, k} ^{n},\left.\widehat{J}_{e x}\right|_{i+1 / 2, j, k} ^{n+1}}^{n+1 / 2} \\
& \approx \rho_{\left.E_{x}\right|_{i+1 / 2, j, k} ^{n},\left.\widehat{J}_{e x}\right|_{i+1 / 2, j, k} ^{n}}^{n+1 / 2, j, k}
\end{aligned}
$$

and applying (12) and rearranging, the resulting equation from taking the variance of (8) yields

$$
\begin{gathered}
\sigma\left\{\left.E_{x}\right|_{i+1 / 2, j, k} ^{n+1}\right\}+\frac{1}{2} \sigma\left\{\left.\widehat{J}_{e x}\right|_{i+1 / 2, j, k} ^{n+1}\right\} \\
=\sigma\left\{\left.E_{x}\right|_{i+1 / 2, j, k} ^{n}\right\}-\frac{1}{2} \sigma\left\{\left.\widehat{J}_{e x}\right|_{i+1 / 2, j, k} ^{n}\right\} \\
\left.+\frac{(\Delta t)^{2}}{\epsilon_{0} \mu_{0} \Delta z} \sigma\left(\frac{\Delta \widehat{H}_{z}}{\Delta y}\right)_{i+1 / 2, j, k}^{n+1 / 2}\right\} \\
\left.-\frac{(\Delta t)^{2}}{\epsilon_{0} \mu_{0} \Delta y} \sigma\left(\frac{\Delta \widehat{H}_{y}}{\Delta z}\right)_{i+1 / 2, j, k}^{n+1 / 2}\right\} .
\end{gathered}
$$

Finally, we will work with the $x$-component equation of (9). Rearranging (9) and taking its variance yields

$$
\begin{aligned}
\sigma^{2}\left\{\frac{\left(\omega_{P e} \Delta t\right)^{2}}{2}\left[\left.E_{x}\right|_{i+1 / 2, j, k} ^{n+1}+\left.E_{x}\right|_{i+1 / 2, j, k} ^{n}\right]\right\} \\
=\sigma^{2}\left\{\left.\left(1+\frac{v_{e} \Delta t}{2}\right) \widehat{J}_{e x}\right|_{i+1 / 2, j, k} ^{n+1}-\left.\left(1-\frac{v_{e} \Delta t}{2}\right) \widehat{J}_{e x}\right|_{i+1 / 2, j, k} ^{n}\right. \\
\quad+\left.\frac{\omega_{c e z} \Delta t}{2} \widehat{J}_{e y}\right|_{i+1 / 2, j, k} ^{n+1}+\left.\frac{\omega_{c e z} \Delta t}{2} \widehat{J}_{e y}\right|_{i+1 / 2, j, k} ^{n} \\
\left.\quad-\left.\frac{\omega_{c e y} \Delta t}{2} \widehat{J}_{e z}\right|_{i+1 / 2, j, k} ^{n+1}-\left.\frac{\omega_{c e y} \Delta t}{2} \widehat{J}_{e z}\right|_{i+1 / 2, j, k} ^{n}\right\} .
\end{aligned}
$$

Apply the following approximations:

$$
\begin{aligned}
& \rho_{\left.E_{x}\right|_{i+1 / 2, j, k} ^{n+1},\left.E_{x}\right|_{i+1 / 2, j, k} ^{n}}^{n} \approx \rho_{\left.\widehat{J}_{e x}\right|_{i+1 / 2, j, k} ^{n+1},\left.\widehat{J}_{e x}\right|_{i+1 / 2, j, k} ^{n}} \\
& \approx \rho_{\left.\widehat{J}_{e y}\right|_{i+1 / 2, j, k} ^{n+1}, \widehat{J}_{e y}}^{n}{ }_{i+1 / 2, j, k} \\
& \approx \rho_{\left.\widehat{J}_{e z}\right|_{i+1 / 2, j, k} ^{n+1},\left.\widehat{J}_{e z}\right|_{i+1 / 2, j, k} ^{n}} \approx 1 \\
& \rho_{\left.\left.\widehat{J}_{e x}\right|_{i+1 / 2, j, k} ^{n \text { and } n+1,} \widehat{J}_{e y}\right|_{i+1 / 2, j, k} ^{n a n d n+1}} \approx \rho_{\left.\widehat{J}_{e x}\right|_{i+1 / 2, j, k} ^{n a n d},\left.\widehat{J}_{e z}\right|_{i+1 / 2, j, k} ^{n a n d}+1}^{n+1} \\
& \approx \rho_{\left.\widehat{J}_{e y}\right|_{i+1 / 2, j, k} ^{n \text { and } n+1},\left.\widehat{J}_{e z}\right|_{i+1 / 2, j, k} ^{n \text { and } n+1}} \approx 1 .
\end{aligned}
$$

From (13) and setting $\left.E_{x}\right|_{i+1 / 2, j, k} ^{n+1}+\left.E_{x}\right|_{i+1 / 2, j, k} ^{n}=E_{x}$, the left-hand side is computed using delta method, yielding

$$
\begin{aligned}
\sigma^{2} & \left\{\frac{\left(\omega_{P e} \Delta t\right)^{2}}{2} E_{x}\right\} \\
\approx & \left(\omega_{P e} \Delta t^{2}\right)^{2} E_{x}^{2} \sigma^{2}\left\{\omega_{P e}\right\}+\left(\frac{\omega_{P e}^{2} \Delta t^{2}}{2}\right)^{2} \sigma^{2}\left\{E_{x}\right\} \\
& +2 \omega_{P e} \Delta t^{2} \frac{\omega_{P e}^{2} \Delta t^{2}}{2} E_{x} \rho_{\omega_{P e}, E_{x}} \sigma\left\{\omega_{P e}\right\} \sigma\left\{E_{x}\right\} \\
= & \left(\frac{\omega_{P e}^{2} \Delta t^{2}}{2}\right)^{2} \sigma^{2}\left\{E_{x}\right\} \\
& +2 \omega_{P e} \Delta t^{2} \frac{\omega_{P e}^{2} \Delta t^{2}}{2} E_{x} \rho_{\omega_{P e}, E_{x}} \sigma\left\{\omega_{P e}\right\} \sigma\left\{E_{x}\right\} \\
& +\left(\omega_{P e} \Delta t^{2}\right)^{2} E_{x}^{2} \rho_{\omega_{P e}, E_{x}}^{2} \sigma^{2}\left\{\omega_{P e}\right\} \\
& -\left(\omega_{P e} \Delta t^{2}\right)^{2} E_{x}^{2} \rho_{\omega_{P e}, E_{x}}^{2} \sigma^{2}\left\{\omega_{P e}\right\}+\left(\omega_{P e} \Delta t^{2}\right)^{2} E_{x}^{2} \sigma^{2}\left\{\omega_{P e}\right\} \\
= & \left(\frac{\omega_{P e}^{2} \Delta t^{2}}{2} \sigma\left\{E_{x}\right\}+\omega_{P e} \Delta t^{2} \rho_{\omega_{P e}, E_{x}} \sigma\left\{\omega_{P e}\right\} E_{x}\right)^{2} \\
& +\left(\omega_{P e} \Delta t^{2}\right)^{2} E_{x}^{2} \sigma^{2}\left\{\omega_{P e}\right\}\left(1-\rho_{\omega_{P e}, E_{x}}^{2}\right) .
\end{aligned}
$$


Neglecting the remainder term $\left(\omega_{P e} \Delta t^{2}\right)^{2} E_{x}^{2} \sigma^{2}\left\{\omega_{P e}\right\}$ $\times\left(1-\rho_{\omega_{P e}, E_{x}}^{2}\right)$, we then have

$$
\begin{aligned}
\sigma^{2}\left\{\frac{\left(\omega_{P e} \Delta t\right)^{2}}{2} E_{x}\right\} \\
\quad=\left(\frac{\omega_{P e}^{2} \Delta t^{2}}{2} \sigma\left\{E_{x}\right\}+\omega_{P e} \Delta t^{2} \rho_{\omega_{P e}, E_{x}} \sigma\left\{\omega_{P e}\right\} E_{x}\right)^{2} .
\end{aligned}
$$

Since $\sigma\left\{E_{x}\right\}=\sigma\left\{\left.E_{x}\right|_{i+1 / 2, j, k} ^{n+1}\right\}+\sigma\left\{\left.E_{x}\right|_{i+1 / 2, j, k} ^{n}\right\}$, we arrive at

$$
\begin{aligned}
& \frac{\left(\omega_{P e} \Delta t\right)^{2}}{2}\left[\sigma\left\{\left.E_{x}\right|_{i+1 / 2, j, k} ^{n+1}\right\}+\sigma\left\{\left.E_{x}\right|_{i+1 / 2, j, k} ^{n}\right\}\right] \\
& +\omega_{P e} \Delta t^{2} \rho_{\omega_{P e}, E_{x}} \sigma\left\{\omega_{P e}\right\}\left(\left.E_{x}\right|_{i+1 / 2, j, k} ^{n+1}+\left.E_{x}\right|_{i+1 / 2, j, k} ^{n}\right) \\
& =\left(1+\frac{v_{e} \Delta t}{2}\right) \sigma\left\{\left.\widehat{J}_{e x}\right|_{i+1 / 2, j, k} ^{n+1}\right\} \\
& -\left(1-\frac{v_{e} \Delta t}{2}\right) \sigma\left\{\left.\widehat{J}_{e x}\right|_{i+1 / 2, j, k} ^{n}\right\}+\frac{\omega_{c e z} \Delta t}{2} \sigma\left\{\left.\widehat{J}_{e y}\right|_{i+1 / 2, j, k} ^{n+1}\right\} \\
& +\frac{\omega_{c e z} \Delta t}{2} \sigma\left\{\left.\widehat{J}_{e y}\right|_{i+1 / 2, j, k} ^{n}\right\}-\frac{\omega_{c e y} \Delta t}{2} \sigma\left\{\left.\widehat{J}_{e z}\right|_{i+1 / 2, j, k} ^{n+1}\right\} \\
& -\frac{\omega_{c e y} \Delta t}{2} \sigma\left\{\left.\widehat{J}_{e z}\right|_{i+1 / 2, j, k} ^{n}\right\} .
\end{aligned}
$$

Rearranging, the resulting equation from taking the variance of (9) yields

$$
\begin{aligned}
- & \frac{\left(\omega_{P e} \Delta t\right)^{2}}{2} \sigma\left\{\left.E_{x}\right|_{i+1 / 2, j, k} ^{n+1}\right\}+\left(1+\frac{v_{e} \Delta t}{2}\right) \sigma\left\{\left.\widehat{J}_{e x}\right|_{i+1 / 2, j, k} ^{n+1}\right\} \\
& +\frac{\omega_{c e z} \Delta t}{2} \sigma\left\{\left.\widehat{J}_{e y}\right|_{i+1 / 2, j, k} ^{n+1}\right\}-\frac{\omega_{c e y} \Delta t}{2} \sigma\left\{\left.\widehat{J}_{e z}\right|_{i+1 / 2, j, k} ^{n+1}\right\} \\
= & \left.\omega_{P e} \Delta t^{2} \rho_{\omega_{P e}, E_{x}} \sigma\left\{\omega_{P e}\right\} E_{x}\right|_{i+1 / 2, j, k} ^{n+1} \\
& +\left.\omega_{P e} \Delta t^{2} \rho_{\omega_{P e}, E_{x}} \sigma\left\{\omega_{P e}\right\} E_{x}\right|_{i+1 / 2, j, k} ^{n} \\
& +\frac{\left(\omega_{P e} \Delta t\right)^{2}}{2} \sigma\left\{\left.E_{x}\right|_{i+1 / 2, j, k} ^{n}\right\} \\
& +\left(1-\frac{v_{e} \Delta t}{2}\right) \sigma\left\{\left.\widehat{J}_{e x}\right|_{i+1 / 2, j, k} ^{n}\right\} \\
& -\frac{\omega_{c e z} \Delta t}{2} \sigma\left\{\left.\widehat{J}_{e y}\right|_{i+1 / 2, j, k} ^{n}\right\}+\frac{\omega_{c e y} \Delta t}{2} \sigma\left\{\left.\widehat{J}_{e z}\right|_{i+1 / 2, j, k} ^{n}\right\} .
\end{aligned}
$$

Although only the $x$-component equations are derived here, analogous equations can be obtained for the $y$-component and $z$-component equations.

Since both the electric field $E_{x}$ and its deviation $\sigma\left(E_{x}\right)$ are in (16), all of the state variables for the deviations of $E$ and $J_{e}$ will depend on the mean of electric field $E$ as well. All of the required equations can be combined to 18 scalar equations with 18 state variables. After the discretization of these equations using $E-J$ collocation, these equations can be divided into three groups. The first group consists of six difference equations for the magnetic field $H$ and its deviation $\sigma(H)$ update similar to
(7) and (14) given for $H_{x}$. The second group consists of six linear equations of all the components of $E, J$, which can be given as

$$
\left[\begin{array}{c}
\left.E_{x}\right|_{i+1 / 2, j, k} ^{n+1} \\
\left.E_{y}\right|_{i, j+1 / 2, k} ^{n+1} \\
\left.E_{z}\right|_{i, j, k+1 / 2} ^{n+1} \\
\left.\widehat{J}_{x}\right|_{i+1 / 2, j, k} ^{n+1} \\
\left.\widehat{J}_{y}\right|_{i, j+1} ^{n+1} \\
\left.\widehat{J}_{z}\right|_{i, j, k+1} ^{n+1} \\
n+1,2
\end{array}\right]
$$

$$
=\left[A^{-1} B\right]\left[\begin{array}{c}
E_{x}^{n} \\
E_{y}^{n} \\
E_{z}^{n} \\
\widehat{J}_{x}^{n} \\
\widehat{J}_{y}^{n} \\
\widehat{J}_{z}^{n}
\end{array}\right]+\left[A^{-1} C\right]\left[\begin{array}{c}
\left(\frac{\Delta \widehat{H}_{z}}{\Delta y}\right)^{n+1 / 2} \\
\left(\frac{\Delta \widehat{H}_{y}}{\Delta z}\right)^{n+1 / 2} \\
\left(\frac{\Delta \widehat{H}_{x}}{\Delta z}\right)^{n+1 / 2} \\
\left(\frac{\Delta \widehat{H}_{z}}{\Delta x}\right)^{n+1 / 2} \\
\left(\frac{\Delta \widehat{H}_{y}}{\Delta x}\right)^{n+1 / 2} \\
\left(\frac{\Delta \widehat{H}_{x}}{\Delta y}\right)^{n+1 / 2}
\end{array}\right] .
$$

Finally, the third group consists of six linear equations of all the components of $\sigma(E)$ and $\sigma(J)$, which can be given as

$$
\begin{aligned}
& {\left[\begin{array}{c}
\sigma\left\{\left.E_{x}\right|_{i+1 / 2, j, k} ^{n+1}\right\} \\
\sigma\left\{\left.E_{y}\right|_{i, j+1 / 2, k} ^{n+1}\right\} \\
\sigma\left\{\left.E_{z}\right|_{i, j, k+1 / 2} ^{n+1}\right\} \\
\sigma\left\{\left.\widehat{J}_{x}\right|_{i+1 / 2, j, k} ^{n+1}\right\} \\
\sigma\left\{\left.\widehat{J}_{y}\right|_{i, j+1 / 2, k} ^{n+1}\right\} \\
\sigma\left\{\left.\widehat{J}_{z}\right|_{i, j, k+1 / 2} ^{n+1}\right\}
\end{array}\right]=\left[A^{-1} B\right]\left[\begin{array}{c}
\sigma\left\{E_{x}^{n}\right\} \\
\sigma\left\{E_{y}^{n}\right\} \\
\sigma\left\{E_{z}^{n}\right\} \\
\sigma\left\{\widehat{J}_{x}^{n}\right\} \\
\sigma\left\{\widehat{J}_{y}^{n}\right\} \\
\sigma\left\{\widehat{J}_{z}^{n}\right\}
\end{array}\right]} \\
& +\left[A^{-1} C\right]\left[\begin{array}{c}
\sigma\left\{\left(\frac{\Delta \widehat{H}_{z}}{\Delta y}\right)^{n+1 / 2}\right\} \\
\sigma\left\{\left(\frac{\Delta \widehat{H}_{y}}{\Delta z}\right)^{n+1 / 2}\right\} \\
\sigma\left\{\left(\frac{\Delta \widehat{H}_{x}}{\Delta z}\right)^{n+1 / 2}\right\} \\
\sigma\left\{\left(\frac{\Delta \widehat{H}_{z}}{\Delta x}\right)^{n+1 / 2}\right\} \\
\sigma\left\{\left(\frac{\Delta \widehat{H}_{y}}{\Delta x}\right)^{n+1 / 2}\right\} \\
\sigma\left\{\left(\frac{\Delta \widehat{H}_{x}}{\Delta y}\right)^{n+1 / 2}\right\}
\end{array}\right]+\left[A^{-1} D\right]\left[\begin{array}{c}
E_{x}^{n+1} \\
E_{y}^{n+1} \\
E_{z}^{n+1} \\
E_{x}^{n} \\
E_{y}^{n} \\
E_{z}^{n}
\end{array}\right]
\end{aligned}
$$

where $A[6 \times 6], B[6 \times 6], C[6 \times 6]$, and $D[6 \times 6]$ are the coefficient matrixes that depend on the plasma properties and the modeling parameters [(19)-(22) at the bottom of the next page]. Note that in this derivation, $E-J$ collocation is utilized such that $\{E, J, \sigma(E), \sigma(J)\}$ are all at the same locations, 


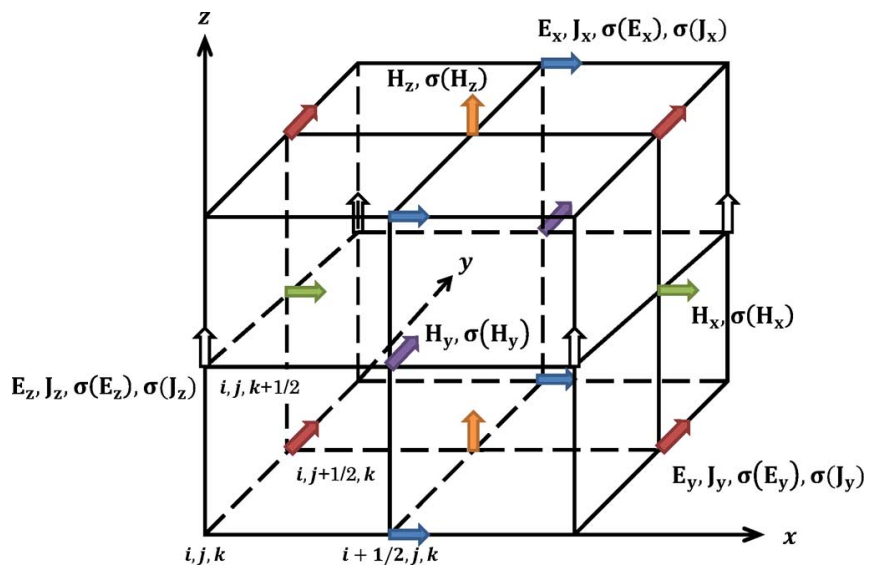

Fig. 1. Yee cell (including stochastic variables) indicating the spatial positioning of the field components.

and $\{H, \sigma(H)\}$ are located at the same positions, as shown in Fig. 1.

Fig. 2 shows a diagram of the iteration process for each time step. What is changed from the regular FDTD update is the addition of the calculation of the variances after the mean values are obtained. In addition, since both the mean fields and their variances behave like waves, both require boundary conditions. Thus, the Mur's boundary condition developed for the magne-

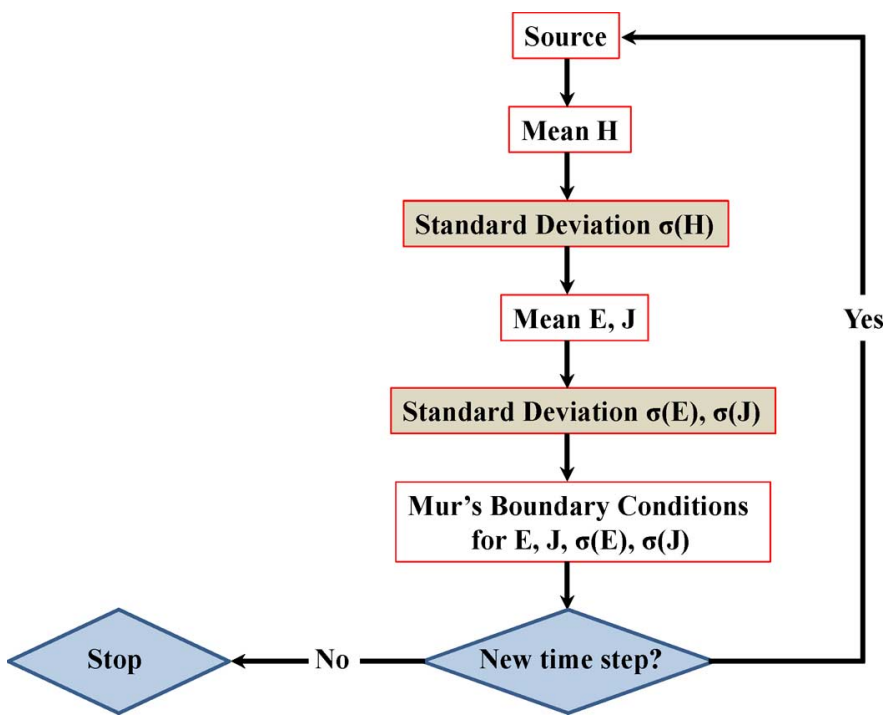

Fig. 2. S-FDTD flowchart.

tized plasma as implemented in [26] is applied to the S-FDTD variance values as well as to the mean values.

\section{VALIDATION OF THE AlgORITHM}

The performance of the fully 3-D S-FDTD cold plasma model of Section II is evaluated by running a similar validation test as

$$
\begin{aligned}
& A=\left[\begin{array}{cccccc}
1 & 0 & 0 & 0.5 & 0 & 0 \\
0 & 1 & 0 & 0 & 0.5 & 0 \\
0 & 0 & 1 & 0 & 0 & 0.5 \\
-\frac{\left(\omega_{P e} \Delta t\right)^{2}}{2} & 0 & 0 & 1+\frac{v_{e} \Delta t}{2} & \frac{\omega_{c e z} \Delta t}{2} & -\frac{\omega_{c e y} \Delta t}{2} \\
0 & -\frac{\left(\omega_{P e} \Delta t\right)^{2}}{2} & 0 & -\frac{\omega_{c e z} \Delta t}{2} & 1+\frac{v_{e} \Delta t}{2} & \frac{\omega_{c e x} \Delta t}{2} \\
0 & 0 & -\frac{\left(\omega_{P e} \Delta t\right)^{2}}{2} & \frac{\omega_{c e y} \Delta t}{2} & -\frac{\omega_{\text {cex }} \Delta t}{2} & 1+\frac{v_{e} \Delta t}{2}
\end{array}\right] \\
& \begin{aligned}
B= & {\left[\begin{array}{cccccc}
1 & 0 & 0 & -0.5 & 0 & 0 \\
0 & 1 & 0 & 0 & -0.5 & 0 \\
0 & 0 & 1 & 0 & 0 & -0.5 \\
\frac{\left(\omega_{P e} \Delta t\right)^{2}}{2} & 0 & 0 & 1-\frac{v_{e} \Delta t}{2} & -\frac{\omega_{c e z} \Delta t}{2} & \frac{\omega_{c e y} \Delta t}{2} \\
0 & \frac{\left(\omega_{P e} \Delta t\right)^{2}}{2} & 0 & \frac{\omega_{c e z} \Delta t}{2} & 1-\frac{v_{e} \Delta t}{2} & -\frac{\omega_{c e x} \Delta t}{2} \\
0 & 0 & \frac{\left(\omega_{P e} \Delta t\right)^{2}}{2} & -\frac{\omega_{c e y} \Delta t}{2} & \frac{\omega_{c e x} \Delta t}{2} & 1-\frac{v_{e} \Delta t}{2}
\end{array}\right] } \\
& {\left[\begin{array}{ccccc}
\frac{(\Delta t)^{2}}{\epsilon_{0} \mu_{0} \Delta z} & -\frac{(\Delta t)^{2}}{\epsilon_{0} \mu_{0} \Delta y} & 0 & 0 & 0
\end{array}\right] }
\end{aligned} \\
& C=\left[\begin{array}{cccccc}
\frac{(\Delta t)^{2}}{\epsilon_{0} \mu_{0} \Delta z} & -\frac{(\Delta t)^{2}}{\epsilon_{0} \mu_{0} \Delta y} & 0 & 0 & 0 & 0 \\
0 & 0 & \frac{(\Delta t)^{2}}{\epsilon_{0} \mu_{0} \Delta x} & -\frac{(\Delta t)^{2}}{\epsilon_{0} \mu_{0} \Delta z} & 0 & 0 \\
0 & 0 & 0 & 0 & \frac{(\Delta t)^{2}}{\epsilon_{0} \mu_{0} \Delta y} & -\frac{(\Delta t)^{2}}{\epsilon_{0} \mu_{0} \Delta x} \\
0 & 0 & 0 & 0 & 0 & 0 \\
0 & 0 & 0 & 0 & 0 & 0 \\
0 & 0 & 0 & 0 & 0 & 0
\end{array}\right] \\
& D=\left[\begin{array}{cccccc}
0 & 0 & 0 & 0 & 0 & 0 \\
0 & 0 & 0 & 0 & 0 & 0 \\
0 & 0 & 0 & 0 & 0 & 0 \\
\omega_{P e} \Delta t^{2} \sigma\left\{\omega_{P e}\right\} & 0 & 0 & \omega_{P e} \Delta t^{2} \sigma\left\{\omega_{P e}\right\} & 0 & 0 \\
\times \rho_{\omega_{P e}, E_{x}} & \omega_{P e} \Delta t^{2} \sigma\left\{\omega_{P e}\right\} & 0 & \times \rho_{\omega_{P e}, E_{x}} & \omega_{P e} \Delta t^{2} \sigma\left\{\omega_{P e}\right\} & 0 \\
0 & \times \rho_{\omega_{P e}, E_{y}} & 0 & \times \rho_{\omega_{P e}, E_{y}} & 0 \\
0 & 0 & \omega_{P e} \Delta t^{2} \sigma\left\{\omega_{P e}\right\} & 0 & 0 & \omega_{P e} \Delta t^{2} \sigma\left\{\omega_{P e}\right\} \\
0 & \times \rho_{\omega_{P e}, E_{z}} & 0 & 0 \rho_{\omega_{P e}, E_{z}}
\end{array}\right] .
\end{aligned}
$$




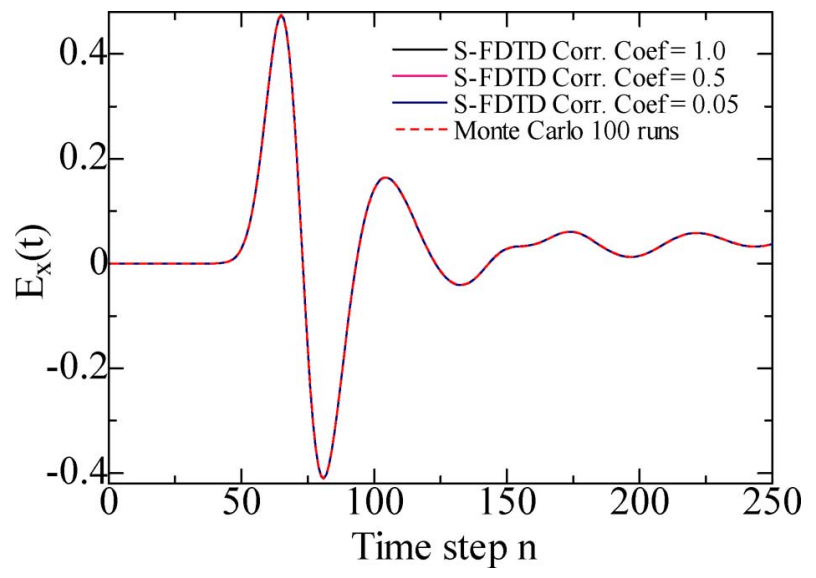

Fig. 3. Mean of $E_{x}$ (observed at a point ten cells away from the source).

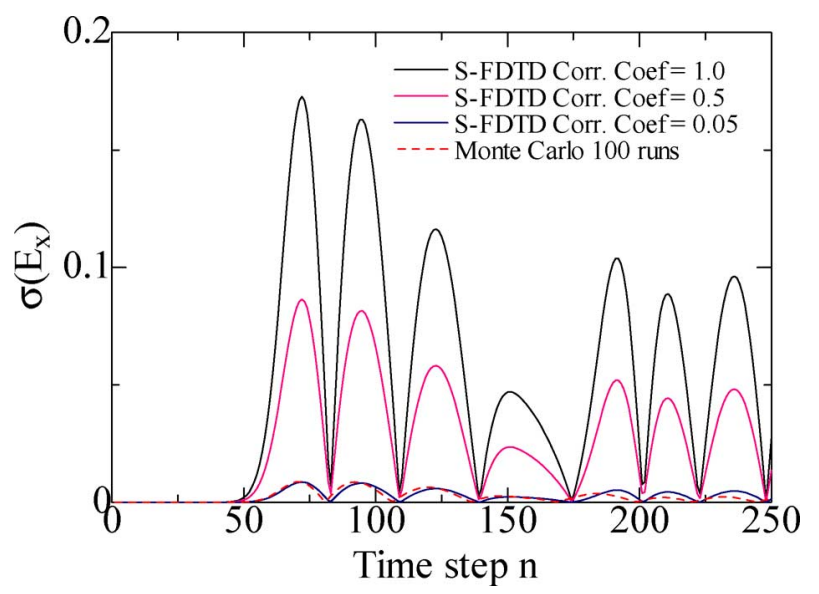

Fig. 4. Standard deviation of $E_{x}$ (observed at a point ten cells away from the source).

for the FDTD plasma model of [17]. An $x$-polarized, $z$-directed Gaussian-pulsed plane wave is modeled using

$$
E_{x}=\exp \left[\frac{-(t-50 \Delta t)^{2}}{2(7 \Delta t)^{2}}\right] .
$$

The lattice space increments in each Cartesian direction of the grid are $\Delta x=\Delta y=\Delta z=1 \mathrm{~mm}$, the time step $\Delta t=$ $\Delta x /(c \times 0.55)$. The plasma medium has a mean electron density $\mu_{n_{e}}=1.0 \times 10^{18} \mathrm{~m}^{-3}$, and the assumed electron density has a statistical variation given by $\sigma\left\{n_{e}\right\}=2.0 \times 10^{16} \mathrm{~m}^{-3}$ (equivalently, $\% \sigma\left\{n_{e}\right\}=2.0$ ). A constant magnetic field $B$ $=0.06 T$ is applied to the plasma as for the validation tests of [17]. For simplicity, a collisionless plasma is tested. This large magnetic field value (relative to the geomagnetic field as would be applied to the ionosphere) is used so that we can see a strong effect of the plasma over a short propagation distance and permit a smaller simulation. The $E_{x}$-field component shown in Fig. 3 is recorded ten cells away from the source in the $z$-direction. The standard deviation and the variance are both important parameters that measure the spread of the distribution about the mean. The standard deviation and variance of the $E_{x}$ field are shown in Figs. 4 and 5, respectively.

For validation, 100 Monte Carlo simulations is used to predict the exact mean, standard deviation, and variance of the fields.

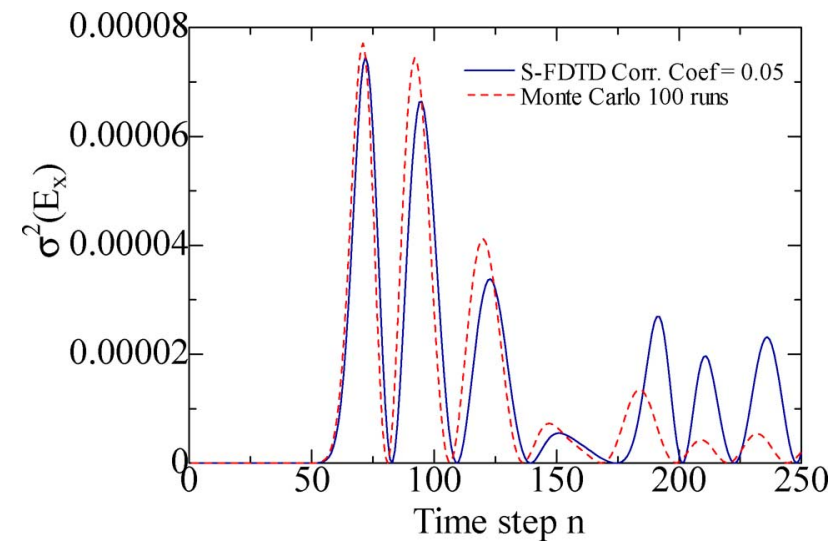

Fig. 5. Variance of $E_{x}$ (observed at a point ten cells away from the source).

The input electron densities $n_{e}$ for each simulation are generated in a random manner with a normal distribution given by

$$
f\left(n_{e}\right)=\frac{1}{\sqrt{2 \pi \sigma^{2}\left\{n_{e}\right\}}} e^{\left\{-\left(n_{e}-\mu_{n_{e}}\right)^{2} / 2 \sigma^{2}\left\{n_{e}\right\}\right\}} .
$$

All of the simulation responses are collected and analyzed to obtain their statistical properties (mean, standard deviation, and variance values). Then, using S-FDTD, three separate simulation cases are run using approximations for the correlation coefficients between the plasma frequency and the electric fields of $1,0.5$, and 0.05 , respectively.

In Fig. 3, there is a strong agreement between the mean values of the Monte Carlo and S-FDTD results. That is, it is found that evaluating the mean values using numbers generated by the random generator is consistent with the mean values obtained directly from the Maxwell's equations/current equation solutions. Fig. 4 shows that a higher correlation coefficient leads to a higher standard deviation (or variance) of the electric field. As expected, the approximations for the cross correlation of the plasma frequency and the electric fields have a direct impact on the accuracy of the S-FDTD method. The correlation coefficient of 1.0 yields a maximum (upper bound) of the variance. In this dataset, a cross-correlation value of 0.05 provides the best agreement with the Monte Carlo simulations.

It is challenging to decide which method should be used to evaluate the cross-correlation coefficients. So far, we have considered the correlation coefficients between the plasma frequency and each of the three components of the $E$ field to be equal. Now instead, Fig. 6 shows results for cases wherein the correlation coefficients are not identical for each of the three Cartesian directions. For example, if we keep $\rho_{\omega, E_{x}}=0.05$ and change $\rho_{\omega, E_{y}}$ and $\rho_{\omega, E_{z}}$ to 0 , Fig. 6 clearly shows that the variance of the $E$ field mainly depends on $\rho_{\omega, E_{x}}$. This can be explained as follows: For an $x$-polarized, $z$-directed plane wave, the $E_{z}$-component is always equal to zero with any value of electron density. The $\rho_{\omega, E_{z}}$, therefore, should also be equal to zero, since a variable and a constant are always independent. Moreover, when the $E_{x}$-component is much larger than the $E_{y}$-component, the value of $\rho_{\omega, E_{x}}$ primarily affects the variance of the field. Note that for a wave that is undergoing 


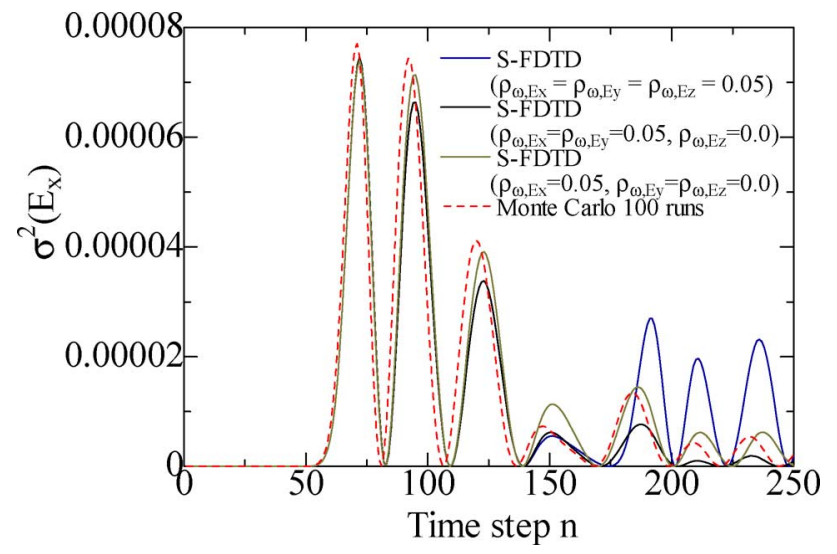

Fig. 6. Variance of $E_{x}$ with changing $\rho_{\omega, E_{y}}$ and $\rho_{\omega, E_{z}}$ (observed at a point ten cells away from the source).

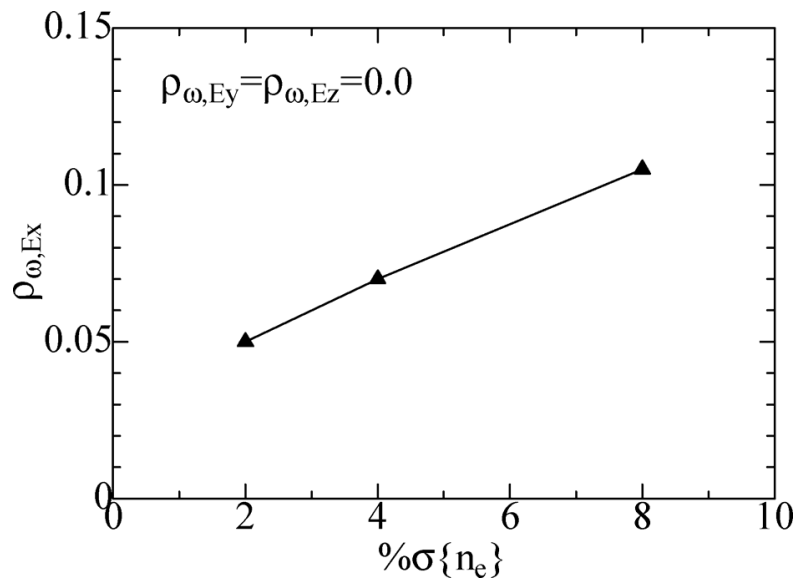

Fig. 7. Correlation coefficient $\rho_{\omega, E_{x}}$ with changing standard deviation of $n_{e}$ (observed at a point ten cells away from the source).

Faraday rotation, the $E_{x}$ - and $E_{y}$-component amplitudes could change with propagation distance. It appears that there are many factors in choosing the best $\rho_{\omega, E}$ values, such as the field component orientation, the cell's location relative to the source, the type of source wave, and the direction of the background magnetic field.

Next, the $\% \sigma\left\{n_{e}\right\}$ is gradually increased from 2 to 8 . Fig. 7 graphs the resulting change in the $\rho_{\omega, E_{x}}$ required for the S-FDTD simulations in order to provide a good agreement with Monte Carlo results. We can see that there is a linear relationship between the choice of correlation coefficients for this range of $\% \sigma\left\{n_{e}\right\}$. We also observe similar trends when changing other input parameters such as the source or mean value of the electron density.

The results shown in Fig. 7 provide an indication as to how to set the cross-correlation coefficient values in the S-FDTD code. That is, two Monte Carlo simulations along with the linear property observed in Fig. 7 can be used to predict the correlation coefficients for an S-FDTD model having an ionosheric plasma at least within the weak scattering range (having, for example, $\% \sigma$ $\left\{n_{e}\right\}=1$ and 2). Note that a relatively small number of simulations is needed for the Monte Carlo method to obtain reasonably accurate results in the cases wherein the standard deviation is small. In contrast, the Monte Carlo method for validating the linear property of the S-FDTD algorithm in the cases of large standard deviation presents a much more significant challenge. More specifically, in a Monte Carlo simulation, the standard error of the mean of the distribution is

$$
\text { error }_{\text {mean }}=\frac{Z \times \sigma_{p}}{\sqrt{\text { runs }}}
$$

where $Z$ is the confidence multiplier of a two-tailed normal distribution. For a $95 \%$ confidence, $Z=2$. For $99 \%, Z=$ 3 , etc. $\sigma_{p}$ is the portfolio's standard deviation. The runs term represents the number of runs in the Monte Carlo simulation. Thus, the error is higher for either a high standard deviation or a small number of runs. Note the accuracy improves as the square root of the ratio of the number of additional runs. As a consequence, tens of thousands of simulations are necessary for Monte Carlo validations when increasing $\% \sigma\left\{n_{e}\right\}$ up to 100 or more. Monte Carlo simulations of 3-D magnetized plasma model for more dense plasma may thus be prohibitively computationally expensive.

As part of future research, systematic studies will be performed to evaluate the best methodology for determining the appropriate correlation coefficients for a wider variety of plasma modeling scenarios. In the S-FDTD methodology of [20] applied to only Maxwell's equations, the correlation coefficients were set to neighboring reflection coefficients. For the modeling presented in this paper, however, there are no reflection surfaces, and so there are no reflection coefficients that can be used for the correlation coefficients. Further, when applied to the ionosphere, the S-FDTD plasma model will use ionospheric parameters that will continuously vary with position and altitude. As a result, a different and more advanced methodology must be followed for determining the appropriate correlation coefficients between the electric field and the plasma frequency. Extensive studies and simulations will be performed as part of future research to develop these best approximations for the correlation coefficients.

In addition, a new and more efficient FDTD magnetized plasma algorithm involving no matrix equations has been recently developed wherein all of the equations are explicit [27]. Using this more efficient algorithm, the gPC method may potentially be applied to electromagnetic-wave propagation in the ionosphere. A gPC-plasma simulation will be derived as part of future work and its results compared with the S-FDTD modeling and Monte Carlo results for validation of the algorithm.

Alternatively, to take advantage of the benefits of the different stochastic modeling approaches, it may possible to create a hybrid method to achieve optimal and efficient results. For example, the gPC method could help the S-FDTD simulations by providing correlation coefficients for cases involving larger deviations of electron density wherein a Monte Carlo validation is extremely inefficient. Note that the computational performance of the S-FDTD method is better than the gPC method since it requires only about twice as much computer simulation time and memory as a traditional FDTD simulation, regardless of numbers of random variables. Overall, the ultimate objective is to develop a stochastic optimization FDTD-based algorithm that 
is well suited for large uncertainty quantification of the ionosphere so that the variability of the EM-wave propagation is well understood.

\section{CONCLUSION}

A 3-D Stochastic FDTD (S-FDTD) model of EM-wave propagation in anisotropic magnetized cold plasma was introduced. The plasma S-FDTD model of this paper is an extension of the S-FDTD model developed by Smith and Furse for Maxwell's equations and applied to biomedical applications [20], [25]. The plasma S-FDTD model of this paper is derived from Maxwell's equations coupled to the current equations derived from the Lorentz equation of motion. When applied to the ionosphere, it uses as input not only average electron (or ion) densities, but also their variance due to uncertainties or variances due to factors such as space weather events.

S-FDTD offers an exceptional improvement in simulation time compared with the brute-force Monte Carlo method. S-FDTD may therefore serve as an important tool for EM ionospheric propagation studies, especially for large 3-D plasma scenarios where Monte Carlo simulations would be impractical to run.

Example S-FDTD simulation results were provided and compared with Monte Carlo results. Different cross-correlation values for the electric fields and the plasma frequency were tested. An upper bound of the variance was obtained by setting these cross-correlation values to 1.0. Future research will be conducted to provide the best methodology for determining these cross-correlation values for different modeling scenarios, particularly for the complex cases of a continuously-varying ionosphere.

\section{ACKNOWLEDGMENT}

The authors would like to thank Dr. S. Smith for technical discussions relating to his S-FDTD model, and the University of Utah's Center for High Performance Computing (CHPC) for providing supercomputing resources.

\section{REFERENCES}

[1] S. C. Aune, "Comparison of ray tracing through ionospheric models," Master's thesis, Dept. of the Air Force, Air Force Inst. of Technol., Wright-Patterson Air Force Base, OH, USA, 2006.

[2] R. Ezquer, M. Mosert, S. Radicella, and C. Jadur, "The study of the electron density variability at fixed heights over San Juan and Tucuman," Adv. Space Res., vol. 29, pp. 993-997, 2002.

[3] M. Mosert, S. Radicella, D. Buresova, R. Ezquerd, and C. Jadur, "Study of the variations of the electron density at $170 \mathrm{~km}$," Adv. Space Res., vol. 29, pp. 937-941, 2002.

[4] M. Mosert, R. Ezquer, C. Jadur, and R. del V. Oviedo, "Time variation of total electron content over Tucumán, Argentina," Geofisica Int., vol. 43, no. 2, pp. 143-151, 2004.

[5] R. Ezquer and M. Mosert, "Ionospheric variability studies in Argentina," Adv. Space Res., vol. 39, pp. 949-961, 2007.

[6] O. Oladipo, J. Adeniyi, S. Radicella, and O. Obrou, "Variability of equatorial ionospheric electron density at fixed heights below the F2 peak," J. Atmos. Solar-Terr. Phys., vol. 70, pp. 1056-1065, 2008.

[7] O. F. Nneka, O. S. Ebere, and H. E. Akpan, "Investigation of electron density variation in some regions of the Ionosphere at Nsukka, Nigeria," Int. J. Library Inf. Sci., vol. 1, pp. 012-016, 2009.

[8] O. A. Oladipo, J. O. Adeniyi, and S. M. Radicella, "Electron density distribution at fixed heights $\mathrm{N}(\mathrm{h})$ : Gaussian distribution test," $J$. Atmos. Solar-Terr. Phys., vol. 71, pp. 1-10, Jan. 2009.
[9] O. Oladipo, J. Adeniyi, S. Radicella, and I. Adimula, "Variability of the ionospheric electron density at fixed heights and validation of IRI2007 profile's prediction at Ilorin," Adv. Space Res., vol. 47, no. 3, pp. 496-505, Feb. 2011.

[10] M. N. Sadiku, Numerical Techniques in Electromagnetics, 2nd ed. Boca Raton, FL, USA: CRC Press, 2001.

[11] K. Yee, "A numerical solution of initial boundary value problems involving Maxwell's equations in isotropic media," IEEE Trans. Antennas Propag., vol. 14, no. 3, pp. 302-307, 1966.

[12] A. Taflove and S. C. Hagness, Computational Electromagnetics: Finite-Difference Time-Domain Method, 3rd ed. Norwood, MA, USA: Artech House, 2005.

[13] S. A. Cummer, "Modeling electromagnetic propagation in the earthionosphere waveguide," IEEE Trans. Antennas Propag., vol. 48, no. 9, pp. $1420-1429,2000$.

[14] M. Thèvenot, J. P. Bérenger, T. Monedière, and F. Jecko, "A FDTD scheme for the computation of VLF-LF propagation in the anisotropic earth-ionosphere waveguide," Ann. Télécommun., vol. 54, pp. 297-310, 1999.

[15] W. Hu and S. A. Cummer, "An FDTD model for low and high altitude lightning-generated EM fields," IEEE Trans. Antennas Propag., vol. 54, no. 5, pp. 1513-1522, 2006.

[16] W. Hu, S. A. Cummer, and W. A. Lyons, "Testing sprite initiation theory using lightning measurements and modeled electromagnetic fields," J. Geophys. Res., vol. 112, pp. 12 645-12 656, 2007.

[17] Y. Yu and J. J. Simpson, "An E-J collocated 3-D FDTD model of electromagnetic wave propagation in magnetized cold plasma," IEEE Trans. Antennas Propag., vol. 58, no. 2, pp. 469-478, 2010.

[18] Y. Yu, "A Three-dimensional FDTD magnetized cold plasma model for global electromagnetic wave propagation," Ph.D. dissertation, Univ. of New Mexico, Albuquerque, NM, USA, 2011.

[19] Y. Yu, J. Niu, and J. J. Simpson, "A 3-D global Earth-ionosphere FDTD model including an anisotropic magnetized plasma ionosphere," IEEE Trans. Antennas Propag., vol. 60, no. 7, pp. 3246-3256, 2012.

[20] S. M. Smith and C. Furse, "Stochastic FDTD for analysis of statistical variation in electromagnetic fields," IEEE Trans. Antennas Propag., vol. 60, no. 7, pp. 3343-3350, Jul. 2012.

[21] T. Tan, A. Taflove, and V. Backman, "Single realization stochastic FDTD for weak scattering waves in biological random media," IEEE Trans. Antennas Propag., vol. 61, no. 2, pp. 818-828, 2013.

[22] R. S. Edwards, A. C. Marvin, and S. J. Porter, "Uncertainty analyses in the finite-difference time-domain method," IEEE Trans. Electromagn. Compat., vol. 52, no. 1, pp. 155-163, Feb. 2010.

[23] A. C. M. Austin and C. D. Sarris, "Efficient analysis of geometrical uncertainty in the FDTD method using polynomial chaos with application to microwave circuits," IEEE Trans. Microw. Theory Techn., vol. 61, no. 12, pp. 4293-4301, Dec. 2013.

[24] G. Casella and R. L. Berger, Statistical Inference, 2nd ed. Pacific Grove, CA, USA: Thompson Learning, 2002.

[25] S. M. Smith, "Stochastic finite-difference time-domain," Ph.D. dissertation, Univ. of Utah, Salt Lake City, UT, USA, 2011.

[26] Y. Yu and J. J. Simpson, "A magnetic field-independent absorbing boundary condition for magnetized cold plasma," IEEE Antennas Wireless Propag. Lett., vol. 10, pp. 294-297, 2011.

[27] A. Samimi and J. J. Simpson, "An efficient 3-D FDTD model of electromagnetic wave propagation in magnetized plasma," IEEE Trans. Antennas Propag., vol. PP, no. 99, 10.1109/TAP.2014.2366203, preprint.

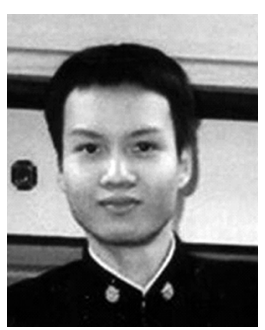

Bach T. Nguyen (S'13) was born in Hanoi, Vietnam. $\mathrm{He}$ received the B.Eng. and M.Eng. degrees in electrical engineering from the National Defense Academy of Japan, Yokosuka, Kanagawa, Japan, in 2007 and 2009, respectively.

From 2010 to 2012, he was with the Vietnam Academy of Science and Technology, Hanoi, Vietnam. Currently, he is working towards the Ph.D. degree in the Electrical and Computer Engineering Department, University of Utah, Salt Lake City, UT, USA. His research interests include computational electromagnetics, RF/microwave technology, RF-IC, and liquid crystal. His current research focuses on stochastic FDTD simulation of electromagnetic wave propagation in ionosphere. 


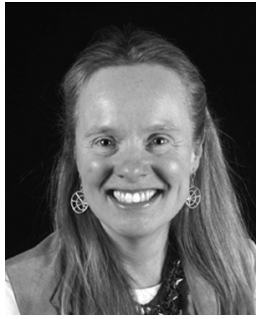

Cynthia Furse (F'08) received the B.S.E.E., M.S.E.E., and Ph.D. E.E. degrees, all from the University of Utah, Salt Lake City, UT, USA, in 1985, 1988, and 1994, respectively.

She is the Associate Vice-President for Research at the University of Utah and Professor in the Electrical and Computer Engineering Department. Her expertise in electromagnetics is applied to sensing and communication in complex lossy scattering media, such as the human body, geophysical prospecting, ionospheric plasma, and aircraft wiring networks. All of these applications have statistical variation, which spawned the research in the present chapter. She has taught electromagnetics, wireless communication, computational electromagnetics, microwave engineering, and antenna design. She also routinely volunteers in Utah's K-12 schools and works with young students to interest them in engineering.

Dr. Furse received the Harriett B. Rigas award for educational excellence from the IEEE in 2009.

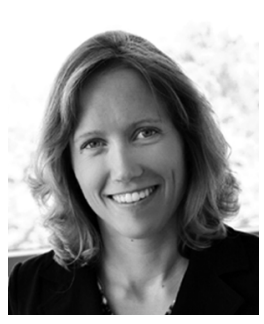

Jamesina J. Simpson (S'01-M'07-SM'12) received the B.S. and Ph.D. degrees in electrical engineering from Northwestern University, Evanston, IL, USA, in 2003 and 2007, respectively.

In 2007, she joined the Electrical and Computer Engineering Department, University of New Mexico, Albuquerque, NM, USA, as a tenure-track Assistant Professor. As of July 2012, she has been an Associate Professor in the Electrical and Computer Engineering Department, University of Utah, Salt Lake City, UT, USA. Her research lab encompasses the application of the full-Maxwell's equations finite-difference time-domain (FDTD) method to model a wide variety of scientific and engineering applications.

Dr. Simpson is a recipient of the National Science Foundation Graduate Research Fellowship and the IEEE AP-S and MTT-S Graduate Research Awards. In 2010, she received an NSF CAREER award, and in 2012 she received the Donald G. Dudley, Jr. Undergraduate Teaching Award of the IEEE AP-S. 